\title{
Decreased expression of repulsive guidance molecule member A by DNA methylation in colorectal cancer is related to tumor progression
}

\author{
ZHI-WEI ZHAO, WEN-JING LIAN, GUO-QING CHEN, HONG-YING ZHOU, \\ GUI-MING WANG, XIA CAO, HUI-JUN YANG and YI-PING HOU \\ Department of Human Anatomy, West China School of Preclinical and Forensic Medicine, \\ Sichuan University, Chengdu 610041, Sichuan Province, P.R. China
}

Received November 11, 2011; Accepted December 28, 2011

DOI: 10.3892/or.2012.1693

\begin{abstract}
Previous studies have shown decreased expression of repulsive guidance molecule member A (RGMa) in colorectal cancer. However, the relationship between the expression levels and promoter DNA methylation status of RGMa and the clinical characteristics of colorectal cancer has not been previously reported. Here, we investigated the expression of RGMa by immunohistochemistry, real-time PCR and western blotting and analyzed the methylation status of the RGMa promoter using Sequenom's MassARRAY platform in colorectal cancer tissues and adjacent normal colorectal tissues. The results showed that RGMa expression was decreased in cancer tissues compared with adjacent normal tissues $(\mathrm{P}<0.01)$. Furthermore, a tendency for decreased expression in tumor tissues was observed from Dukes' stage A to stage $\mathrm{D}(\mathrm{P}<0.01)$. In addition, significantly higher levels of hypermethylation in promoter regions of RGMa were observed in colorectal cancer tissues, compared with those in adjacent normal colorectal tissues $(\mathrm{P}<0.01)$. Moreover, the methylation levels of RGMa in tumor tissues were significantly increased in Dukes' stage C and D compared with Dukes' stage A and $\mathrm{B}(\mathrm{P}<0.01)$. Our results indicate that RGMa expression and promoter methylation status are closely related to colorectal cancer genesis and progression. Determination of the expression level and methylation frequency of RGMa in colorectal cancer tissues may have benefit for early diagnosis and for evaluating patient prognosis.
\end{abstract}

Correspondence to: Professor Yi-Ping Hou or Dr Hui-Jun Yang, Department of Human Anatomy, West China School of Preclinical and Forensic Medicine, Sichuan University, Renmin South Road 17, Chengdu 610041, Sichuan Province, P.R. China

E-mail: yip_hou@126.com

E-mail: he500209@163.com

Key words: repulsive guidance molecule member A, DNA methylation, colorectal cancer, tumor progression

\section{Introduction}

Colorectal cancer is one of the most common cancers in the world. The incidence and mortality rates of colorectal cancer have increased over the last several decades (1). In spite of novel approaches of treatment available today, the prognosis of advanced colorectal cancer is generally very poor. Therefore, it is important to identify the tumor specific molecular markers that might influence survival and may serve as potential therapeutic targets. To date, unfortunately, there are few molecular markers that have been validated to demonstrate clinical utility.

RGMa, a member of the RGM family of membrane-bound proteins was originally isolated as a retinal axon guidance molecule. It is expressed in the neural tube before the appearance of retinal axons, promoting neuronal differentiation and axon guidance (2). Apart from the nervous system, RGMa is also expressed in a wide range of tissues including heart, lung, liver, skin, leukocytes, kidney, testis and the gut $(3,4)$. In the nervous system, RGMa functions as repulsive cure for the navigation of axonal growth through its receptor, neogenin (5-7). In leukocytes, RGMa inhibits leukocyte migration by contact repulsion and chemorepulsion, acting as an endogenous inhibitor of leukocyte chemotaxis (8). In the gastrointestinal tract, RGMa is expressed in the proliferative basal crypt epithelial cells (4), but its function remains unknown. Recent studies have indicated that RGMa is also involved in BMP signaling acting as a co-receptor of BMP (9). RGMa mediates BMP signaling via an interaction with type I receptors and type II receptor, thereby activating the intracellular SMAD1/5/8 cascade $(3,10)$.

In terms of its role in cancer, recent studies have found that RGMa expression is downregulated in colorectal cancer and breast cancer. Moreover, RGMa is closely related to BMP and neogenin signaling, which have been confirmed to be involved in tumor development and progression. Here, we hypothesize that RGMa may be involved in tumorigenesis and progression of colorectal cancer. However, the relationship between the expression of RGMa and the tumor stage and progression have not been reported. Aberrant methylation of promoter $\mathrm{CpG}$ islands is associated with transcriptional silencing of several 
genes, such as p16, E-cadherin and LINE-1, in human cancer $(11,12)$. Previous studies have shown that the downregulation of RGMa in colorectal cancer tissue and cell lines is related to DNA methylation (13). To date, the relationship between the methylation status of RGMa and the stage of colorectal carcinomas is unknown.

In the present study, we detected and compared the expression of RGMa and the methylation status of the RGMa promoter in human colorectal cancer specimens and corresponding normal colorectal tissue. Furthermore, the association of RGMa expression and promoter methylation with different tumor stages was also investigated.

\section{Materials and methods}

Tissue specimens. Forty fresh colorectal tumor tissues and patient-matched adjacent normal colorectal tissues were obtained from patients who underwent surgery at the West China Hospital of the Sichuan University. Adjacent normal colorectal tissues were collected from the area at least $5 \mathrm{~cm}$ from the edge of the tumor. Pathological examination further confirmed that no cancer cells were evident in the collected adjacent normal tissues. Parts of the fresh specimens were frozen in liquid nitrogen and stored at $-80^{\circ} \mathrm{C}$ for total RNA, total DNA and protein isolation. Other parts were fixed in $4 \%$ paraformaldehyde and embedded in paraffin for histological sections and immunohistochemical staining. In addition, eighty paraffin-embedded colorectal tumor tissues were obtained from the Cancer Hospital of Sichuan Province for immunohistochemical staining. Cases were staged according to the Dukes' classification system. None of the patients received chemotherapy or radiotherapy prior to surgery. The study was approved by the local Ethics Standards of the Institutional Review Board. Pathological diagnosis was based on the morphologic microscopic features of tumor cells by $\mathrm{H} \& \mathrm{E}$ staining.

Immunohistochemistry. Immunohistochemical examination was performed on paraffin-embedded tissue sections. The sections were dewaxed in xylene and immersed in graded ethanol series and distilled water. Immunohistochemical staining was performed using the avidin-biotin peroxidase complex (ABC) method, according to the manufacturer's instructions. The primary antibody for RGMa (Abcam) was diluted to 1:200. The primary antibody was omitted as a negative control for the immunostaining. The tissue specimens were viewed separately by two pathologists under double-blind conditions. The immunoreactivity score system has been previously described (14).

RNA isolation and quantitative RT-PCR. Total RNA from 40 cancers and adjacent normal colorectal tissues was extracted using TRIzol (Invitrogen) according to the manufacturer's instructions. The yield of the extracted RNA was determined by measuring the optical density at $260 \mathrm{~nm}$. Total RNA was retrotranscribed using the RevertAid ${ }^{\mathrm{TM}}$ First Strand cDNA Synthesis kit (Fermentas) according to the manufacturer's instructions. PCR amplification and detection of the PCR amplified gene products were performed with the SYBRGreen PCR master mix (Tiangen Biotech). Real-time PCR was performed on the Mastercycler ep realplex (Eppendorf). The reaction cycle consisted of a hot start at $95^{\circ} \mathrm{C}$ for $10 \mathrm{~min}$, then 30 cycles of denaturation at $95^{\circ} \mathrm{C}$ for $30 \mathrm{sec}$ and extension at $65^{\circ} \mathrm{C}$ for $30 \mathrm{sec}$. Levels of mRNA expression were quantified after normalization with endogenous control GAPDH using the $2{ }^{-\triangle \Delta C T}$ method (15). The primer sequences used for PCR were as follows: GAPDH, 5'-ctttggtatcgtggaaggactc-3', 5'-gtagaggcagggatgatgttct-3' (product size, 132 bp); RGMa, 5'-aagggagaggctagtggtaacag-3', 5'-gagttgcacttgaggatcttgc-3' (product size, $153 \mathrm{bp}$ ). Experiments were performed independently for each sample and at least 3 technical replicates were run for each treated sample and controls.

Western blot analysis. For protein extraction, each sample was put into powder in liquid nitrogen with a pre-cooled mortar and pestle. The tissue powder were conducted using lysis buffer (50 mM Tris-HCl, pH 7.4; 150 mM NaCl; 1\% NP-40; $0.25 \%$ sodium deoxycholate; $1 \mathrm{mmol} / 1 \mathrm{EDTA} ; 1 \mathrm{mmol} / 1$ phenylmethlsulfonyl fluoride; $1 \mathrm{mmol} / 1 \mathrm{Na}_{3} \mathrm{VO}_{4}$ and $1 \mathrm{mmol} / \mathrm{l}$ $\mathrm{NaF}$ ) and incubated at $4^{\circ} \mathrm{C}$ for $30 \mathrm{~min}$, followed by centrifugation at $10,000 \mathrm{xg}$ at $4^{\circ} \mathrm{C}$ for $20 \mathrm{~min}$. Protein concentrations were then measured using protein assay kits (Bio-Rad Laboratories). Next, the protein lysates were resolved by SDS-PAGE, and then transferred onto polyvinylidene difluoride membranes (GE Healthcare Biosciences), blocked with PBS containing $0.2 \%$ Tween-20 and 5\% non-fat dry milk, and incubated with a primary antibody against RGMa $(1: 1,000)$ from Abcam. Antibody binding was revealed by incubation with horseradish peroxidase-conjugated secondary antibodies and an ECL Plus immunoblotting detection system (GE Healthcare Biosciences). Signals were quantified using the NIH ImageJ 1.63 Software.

Quantitative DNA methylation analyses. Genomic DNA was extracted from 40 cancers and adjacent normal colorectal tissues by a tissue DNA kit (Qiagen) according to the manufacturer's instructions. The concentration and purity of the DNA were determined by absorbance at 260 and $280 \mathrm{~nm}$. A total of $200 \mathrm{ng}$ genomic DNA from each sample was bisulfite-treated with the Methylamp DNA Modification kit (Epigentek). The quality of the bisulfite conversion was controlled by using PCR products. The Sequenom MassARRAY platform was used to perform the quantitative methylation analysis of RGMa.

This system uses matrix-assisted laser desorption/ionization time-of-flight (MALDI-TOF) mass spectrometry in combination with RNA base-specific cleavage (Mass Cleave). A detectable pattern is then analyzed for its methylation status. We analyzed the RGMa promoter region (Fig. 1A) and selected two fragments in the $\mathrm{CpG}$ islands. The following primers were designed: the first is (5'-aggaagagagAGTTTAGGGAAGGAT TTTTGTTGTG-3' and 3'-cagtaatacgactcactatagggagaaggct AAATTAAAACCACATCTCTAAAAACCA-5') to amplify base pairs -1428 to -1121 of the RGMa promoter (Fig. 1B). The second is (5'-aggaagagagGAGTTTTTTTGAGATTTTTG GAGAA-3' and 3'-cagtaatacgactcactatagggagaaggctACT ATTTCACAACAAAAATCCTTCC-5') to amplify base pairs -1153 to -674 of the RGMa promoter (Fig. 1C). The spectra methylation ratios were generated by EpiTYPER Software version 1.0 (Sequenom, Inc.). 
A

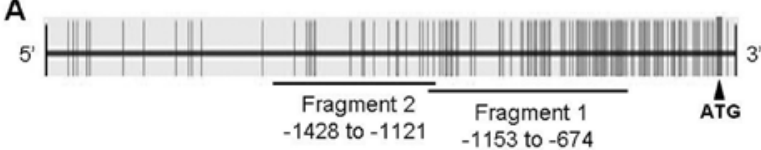

B

5' GAGTTCCTCTGAGATTTCTGGAGAAGGCGGTTACATCCAAGACAAATGCTGTTGC GCTC TGCGGAGACGCACCTTTGTTCTATAGAGATTTTGTTGTTGTTTAAGAATCTTCCTGAAAA AACTAATACATATGTATTACATGCTAACACACG GATACATTCTTGCGTAGAATTGTACAT AACTCAGGAGTGTCTAGAGTGAAAAGACCGTATCGCACTCCCTCCCACGGCCECGGCT

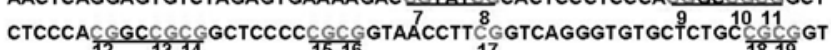
TCTTTCCAGTCTCTG 20 CTITTACATGCACAC 21 CACAGATTG 22 AGTGGAATCTCCTGG GCTTTTCAAAAGAATGTTTTCAACATTAAGC 23 CATGCACACAGTGGCTTGAACACGGCC A $\frac{C G T C G G G A G C A G T A A A T C C C C A G A G G C T C C G G G A G C C C A G G G A A G G A C C C T T G C T G}{25}$ TGAAACAGC

C

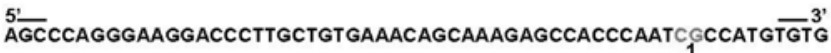
GATCTGAAACCCCAGCTGGAGCZ GTGAAGTCGGTCTGGTTACACAGCTCAGGCCCAAAT TAGCACTGCACGGTTAGAGAGATTTGCCAGTACATTCTAGACAGGGTTATGACCTTCCC CTTCTTACAGAACAAACTGTCTTGAATTTTGATATGATGAGAACTCTGGATTTGCAAAAT GAAAAGGTATCETCGCCATTAAAACACGATCGTTATTTATGCGGTGTGGTCTTCAGAGAT GTGGTTTTAACTC

Figure 1. Schematic diagram of the RGMa promoter and the sequences for the Sequenom MassARRAY platform analysis. (A) Map of the RGMa promoter, positions of $\mathrm{CpG}$ (vertical bars) and fragments for methylation study; (B) the sequence represents a 479 bp fragment (fragment 1, base pairs between -1153 to -674) in the promoter of RGMa; (C) the sequence represents a 307 bp fragment (fragment 2, base pairs between -1428 to -1121) in the promoter of RGMa. The numbers refer to the location of the $\mathrm{CpG}$ sites and the underlinings highlight the $\mathrm{CpG}$ sites including more than one $\mathrm{CpG}$ site tested at the same time.
Statistical analysis. Data were expressed as the mean \pm SD. The paired t-test, one-way ANOVA and Mann Whitney U test and Kruskal-Wallis test were applied for statistical analysis by using the SPSS software (11.0 versions). Statistical significance was set at $\mathrm{P}<0.05$.

\section{Results}

Immunohistochemical staining of RGMa in normal adjacent colorectal tissue and colorectal cancer tissue. In the present study, we performed immunostaining of 120 colorectal tumor samples and 40 adjacent normal colorectal tissue samples. Cellular staining for RGMa was detected in both the membrane and the cytoplasm (Fig. 2). Based on the scoring system of immunostaining, statistical analysis revealed a significant decrease of RGMa expression in colorectal cancers compared with normal tissue $(\mathrm{P}<0.01)$ (Table I). Most importantly, the lower expression of RGMa was closely related with tumor stage (Table I). Moreover, the decrease of RGMa from Dukes' stage A to $\mathrm{D}$ was also significant $(\mathrm{P}<0.01)$. These results indicate that decreased expression of RGMa is associated with tumor progression.

Quantitative analysis of RGMa expression in colorectal cancer tissues and matched normal tissues by real-time PCR and western blot analysis. In our study, we performed quantitative analysis of mRNA and protein expression levels of RGMa in 40 colorectal cancer tissues and matched adjacent
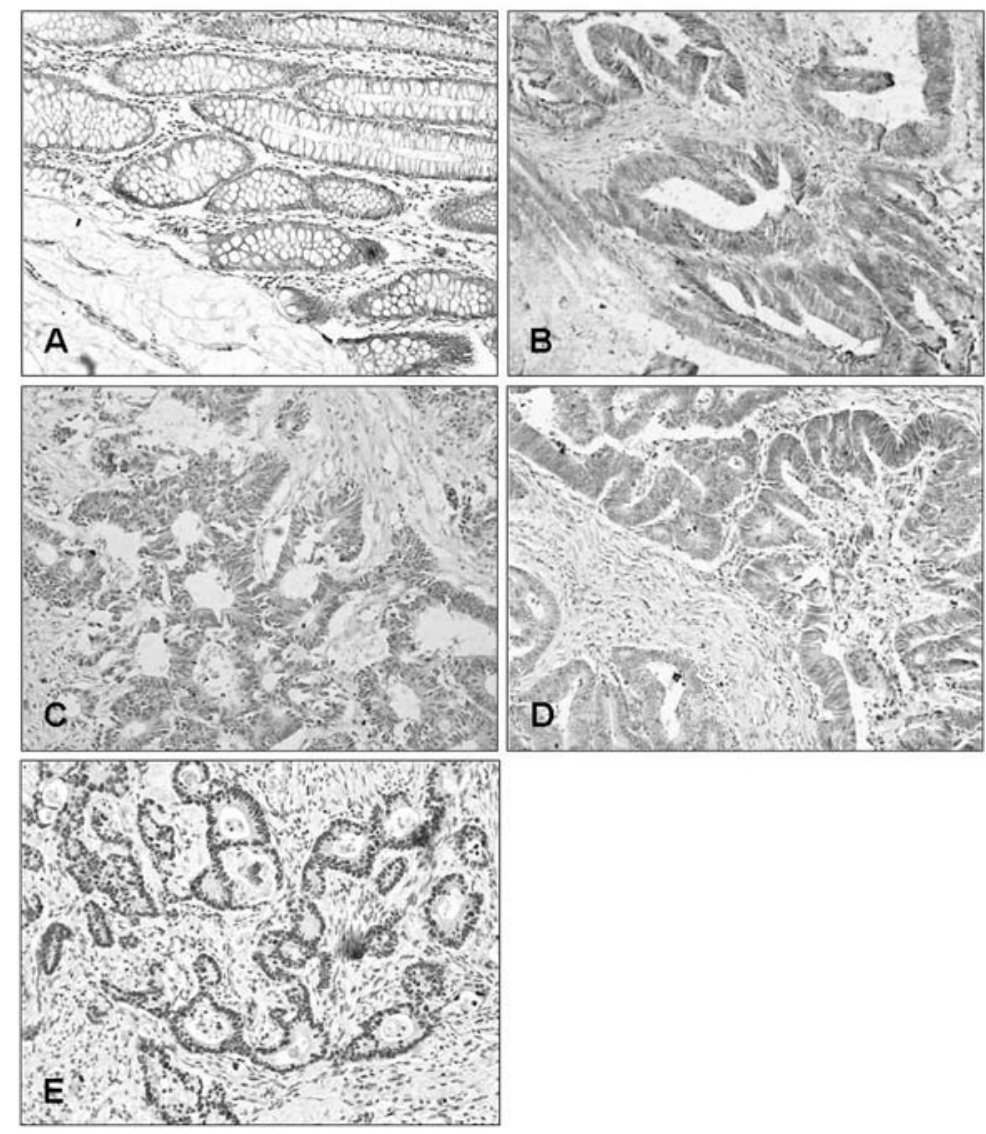

Figure 2. RGMa expression by immunohistochemical staining. (A) Adjacent normal colorectal tissue; colorectal cancer tissue with (B) Dukes' stage A; (C) Dukes' stage B; (D) Dukes' stage C and (E) Dukes' stage D. 
Table I. Relationship between tumor RGMa expression and clinical characteristics.

\begin{tabular}{|c|c|c|c|c|c|c|}
\hline \multirow[b]{2}{*}{ Characteristics } & \multirow[b]{2}{*}{$\mathrm{n}$} & \multicolumn{4}{|c|}{ RGMa expression } & \multirow[b]{2}{*}{$\mathrm{P}$-value } \\
\hline & & - & + & ++ & +++ & \\
\hline Total & 120 & 9 & 33 & 47 & 31 & \\
\hline Gender & & & & & & $0.150^{\mathrm{a}}$ \\
\hline Male & 65 & 4 & 22 & 26 & 13 & \\
\hline Female & 55 & 5 & 11 & 21 & 18 & \\
\hline Age & & & & & & $0.834^{\mathrm{a}}$ \\
\hline$\geq 60$ & 81 & 7 & 22 & 29 & 23 & \\
\hline$<60$ & 39 & 2 & 11 & 18 & 8 & \\
\hline Tumor side & & & & & & $0.073^{\mathrm{b}}$ \\
\hline Ascending colon & 45 & 5 & 8 & 17 & 15 & \\
\hline Transverse colon & 9 & 0 & 3 & 2 & 4 & \\
\hline Descending colon & 3 & 0 & 2 & 1 & 0 & \\
\hline Sigmoid colon & 57 & 4 & 19 & 26 & 8 & \\
\hline Rectum & 6 & 0 & 1 & 1 & 4 & \\
\hline Dukes' stage & & & & & & $0.006^{\mathrm{b}}$ \\
\hline A & 30 & 1 & 8 & 15 & 6 & \\
\hline $\mathrm{B}$ & 30 & 0 & 6 & 11 & 13 & \\
\hline $\mathrm{C}$ & 30 & 3 & 7 & 12 & 8 & \\
\hline $\mathrm{D}$ & 30 & 5 & 12 & 9 & 4 & \\
\hline
\end{tabular}

${ }^{a}$ The Mann-Whitney test was applied; ${ }^{b}$ the Kruskal-Wallis test was applied. P-value in bold denotes significant difference.

A

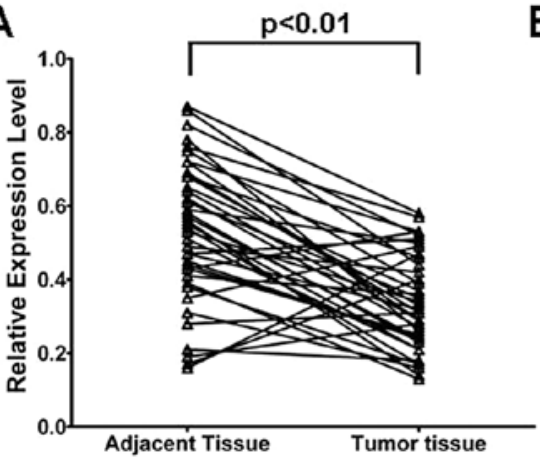

B

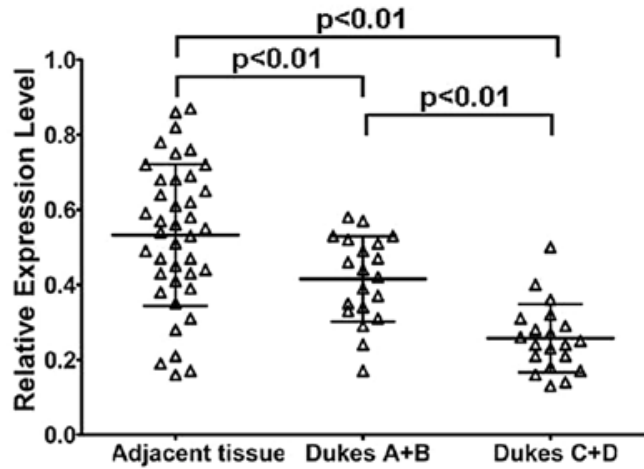

C

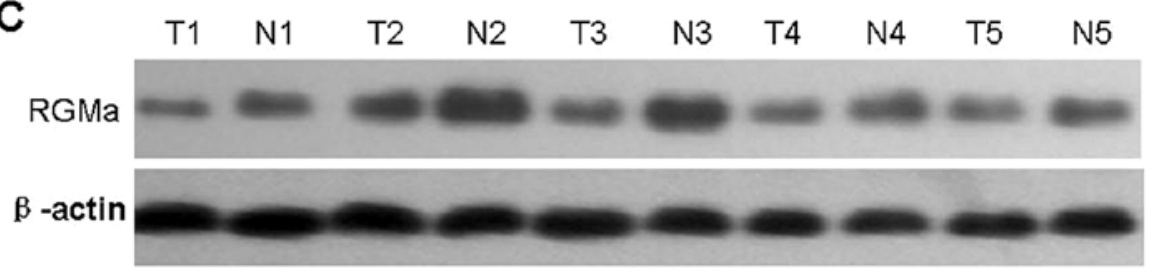

Figure 3. Real-time PCR and western blot analysis analyzed the expression of RGMa in colorectal cancer and adjacent normal colorectal tissues. (A) Quantitative RGMa mRNA expression in paired tumor tissues and adjacent tissues from 40 cases; (B) quantitative analysis of RGMa mRNA in adjacent tissues (n=40), tumor tissue with Dukes' stage A and B ( $n=20)$ and tumor tissue with Dukes' stage C and $D(n=20)$; (C) western blot analysis the RGMa protein expression in tumor tissues and adjacent normal tissues. $\mathrm{T}$, tumor tissue; $\mathrm{N}$, adjacent normal tissue.

normal tissues. Real-time PCR was used to detect the mRNA levels of the RGMa gene. Among the 40 samples of clinical specimens, 33 samples of colorectal cancer tissues showed lower expression than in adjacent normal tissues; 7 samples of colorectal cancer tissues showed higher expression than in adjacent normal tissues. Compared with the normal tissues, RGMa expression was significantly higher in the tumors $(\mathrm{P}<0.01)$ (Fig. 3A). Subsequently, we investigated whether the expression 

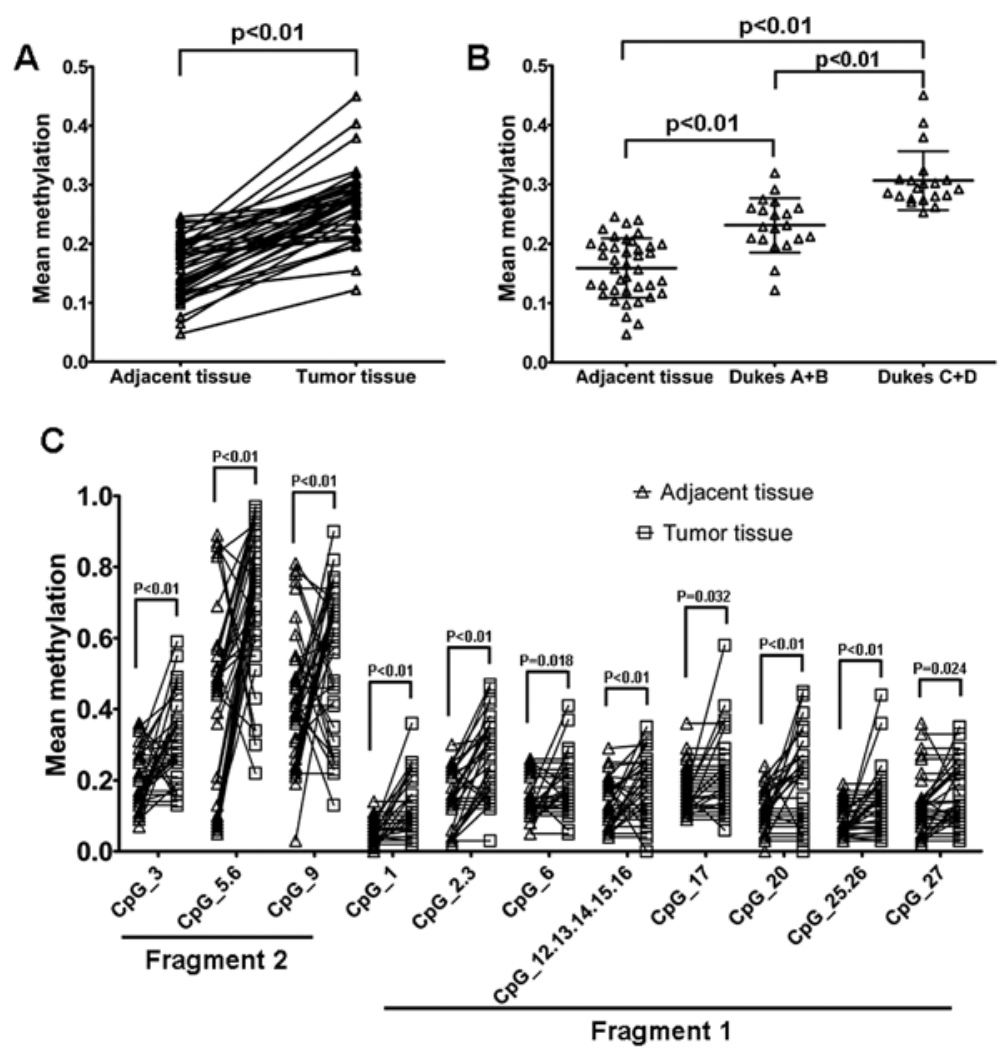

Figure 4. Quantitative analysis of methylation levels in colorectal tumor and adjacent normal colorectal tissues by MassARRAY. (A) Quantitative analysis of RGMa methylation levels in paired tumor tissues and adjacent tissues from 40 cases; (B) quantitative analysis of RGMa methylation level in adjacent tissues $(n=40)$, tumor tissue with Dukes' stage A and B $(n=20)$ and tumor tissue with Dukes' stage C and D $(n=20)$; (C) methylation level of CpG sites in RGMa gene in tumor tissues and adjacent tissues.

of RGMa correlated with tumor progression. Our data showed that RGMa expression gradually decreased from Dukes' stage A to stage $\mathrm{D}$ and there was a significant difference between Dukes' stage $\mathrm{A}+\mathrm{B}$ and stage $\mathrm{C}+\mathrm{D}(\mathrm{P}<0.01)$ (Fig. 3B). As for RGMa protein expression, western blot analysis showed that RGMa protein levels decreased significantly in tumor tissues compared with adjacent normal tissues (Fig. 3C). These results were consistent with the immunohistochemistry results.

DNA hypomethylation in colorectal cancer. The methylation level of CpG sites of the RGMa promoter was evaluated by the Sequenom MassARRAY platform. There were 2 DNA fragments analyzed in our study. In fragment 1 (-1153 to-674), a total of $27 \mathrm{CpG}$ sites were divided into $19 \mathrm{CpG}$ sites (Fig. 1B). In fragment 2 (-1482 to -1121), a total of $9 \mathrm{CpG}$ sites were divided into $7 \mathrm{CpG}$ sites (Fig. 1C). Significantly higher levels of hypermethylation in the promoter regions of RGMa were observed in colorectal cancer tissues, as compared with that in adjacent normal colorectal tissues $(\mathrm{P}<0.01)$ (Fig. 4A). To further determine whether the level of methylation of RGMa was related to tumor progression, we compared the methylation level of RGMa among colorectal cancer tissues from Dukes' stage A to stage D. The results showed that the methylation level of RGMa was significantly higher in Dukes' stage $\mathrm{C}$ and D compared with the Dukes' stage A and B $(\mathrm{P}<0.01)$ (Fig. 4B). These data suggest that hypermethylation in the RGMa gene promoter may be associated with the development and progression of human colorectal cancer.
In addition, we also analyzed the methylation level of each $\mathrm{CpG}$ site in colorectal cancer tissues and normal tissues. Methylation levels varied at different $\mathrm{CpG}$ sites. In normal tissues, the lowest methylation level $(5.45 \%)$ was located at the site 1 in fragment 1, and the highest methylation level (32.13\%) was located at site 9 in fragment 1 . On the other hand, in tumor tissues, the lowest methylation level $(10.73 \%)$ was located at site 1 in fragment 1 and the highest methylation level (71.32\%) was located at sites 5 and 6 in fragment 1. Compared with adjacent normal tissue, dramatic methylation modification was observed in $\mathrm{CpG}$ sites 3,5 6 and 9 in fragment 1 and $\mathrm{CpG}$ sites $1,2,3,12,13,14,15,16,20,25$ and 26 in fragment 2; no significant change in the methylation levels was observed at CpG sites 6, 17 and 27 in fragment 2 (Fig. 4C).

\section{Discussion}

RGMa, a member of the RGM family, was originally discovered as a cell surface protein with key developmental roles in the nervous system (16). In initial studies, RGMa was thought to be an axonal guidance molecule whose function was to regulate cephalic neural tube closure, inhibit neurite outgrowth and cortical neuron branching, and the formation of mature synapses (17-20). Binding to its receptor neogenin, RGMa could induce the activation of the RHOA-ROCK1/phokinase singaling pathway through the UNC5bB-AGHGEF12/ LARG-PTK2 cascade, leading to collapse of the neuronal growth cone and neurite outgrowth inhibition (21). In addition, 
RGMa could also lead to HRAS inactivation by influencing the HRAS1-PTK2-AKT1 signaling pathway (6). Recently, RGMa has been implicated in the BMP signaling pathway acting as a co-recepctor of $\operatorname{BMP}(3,9,22,23)$. BMPs, members of the TGF- $\beta$ superfamily, were originally identified as molecules that induced bone and cartilage formation and are now considered to be multifunctional cytokines, playing an important role in the cancer $(24,25)$. BMPs have been confirmed to be involved in the development and progression of various malignancies, including breast, prostate, lung and colorectal cancer. In colorectal cancer, it has been demonstrated the BMP acts as a tumor suppressor to induce apoptosis and to increase cancer cell response to chemotherapy (26-28). Collectively, the existing data suggest that RGMa has a dual role as both an axonal guidance molecule and a co-receptor of BMP signaling. To date, only a few studies have investigated the expression and function of RGMa in cancer. Li et al reported that RGMa is downregulated by DNA methylation and is involved in cancer proliferation, migration and invasion and in the response to DNA-damaging agents in colorectal cancer (13). Aberrant expression of RGMa has been observed in breast cancer and has been associated with cancer progression and poor prognosis (29). However, the precise function of RGMa in cancer remains unknown. Furthermore, it is still unclear whether the expression level and promoter methylation level of RGMa is related to tumor progression in colorectal cancer.

In the present study, we investigated the expression of RGMa by immunohistochemistry in 80 cases of human colorectal cancer samples. We also analyzed the mRNA and protein levels of RGMa in 40 colorectal cancer tissues and matched normal adjacent tissues. The results showed that RGMa mRNA and protein was abundantly expressed in normal colorectal tissues; RGMa levels decreased in colorectal cancer compared with normal adjacent normal colorectal tissues from the same individual $(\mathrm{P}<0.01)$. Moreover, we demonstrated that RGMa mRNA and protein levels were much higher in patients with Dukes' stage A and B than with the Dukes' C and D. Furthermore, the RGMa expression levels in cancer tissues showed a negative correlation to tumor progression, although RGMa expression was not significantly different between patients with Dukes' C and D. Similar results were obtained by western blot analysis and immunohistochemistry. Because of the close relation between Dukes' staging and prognosis, these results indicate that RGMa may act as a potential marker of colorectal cancer diagnosis and prognosis. This is the first study to show that in colorectal cancer expression of RGMa is related to tumor stage. Whether expression of RGMa is related to tumor cell differentiation and tumor metastasis remains to be elucidated.

Previous studies have confirmed that transcriptional silencing of tumor suppressor genes by aberrant methylation of $\mathrm{CpG}$ islands plays a crucial role in the development of various cancers (30). Many genes involved in the regulation of cell cycle, tissue invasion, DNA repair and apoptosis have been shown to be inactivated by this type of epigenetic mechanism. Consistent with these data, our results from quantitative methylation analysis of RGMa by the Sequenom MassARRAY platform and by real-time PCR also showed the relation between the RGMa methylation status and its expression in colorectal cancer tissue. Many studies have examined the methylation status of specific genes in the progression stage of cancer. In our study, it was confirmed that RGMa methylation occurs in adjacent normal tissue and tumor tissue of colorectal cancer. However, the methylation frequency in the tumor tissue $(26.86 \%)$ was much higher than that in the normal tissue (18.26\%). In tumor tissue, the methylation frequency of RGMa in specimens of Dukes' stage C and D (30.63\%) was higher than those of Dukes' stage A and B $(23.10 \%)$. This result suggests that RGMa methylation increases with the progression of colorectal cancer and is related to the development of colorectal cancer.

In conclusion, the present study revealed that RGMa was abundantly expressed in colorectal tissue and its expression levels were downregulated during tumorigenesis and tumor progression. Moreover, we demonstrated that DNA methylation was involved in the regulation of the expression of RGMa and the methylation frequency of RGMa was closely related to colorectal cancer development and progression. Determination of the expression level and methylation frequency of RGMa in colorectal cancer tissues may assist in early diagnosis and for evaluating patient prognosis.

\section{References}

1. Center MM, Jemal A and Ward E: International trends in colorectal cancer incidence rates. Cancer Epidemiol Biomarkers Prev 18: 1688-1694, 2009.

2. Severyn CJ, Shinde U and Rotwein P: Molecular biology, genetics and biochemistry of the repulsive guidance molecule family. Biochem J 422: 393-403, 2009.

3. Babitt JL, Zhang Y, Samad TA, Xia Y, et al: Repulsive guidance molecule (RGMa), a DRAGON homologue, is a bone morphogenetic protein co-receptor. J Biol Chem 280: 29820-29827, 2005.

4. Metzger M, Conrad S, Alvarez-Bolado G, et al: Gene expression of the repulsive guidance molecules during development of the mouse intestine. Dev Dyn 234: 169-175, 2005.

5. Wilson NH and Key B: Neogenin interacts with RGMa and netrin-1 to guide axons within the embryonic vertebrate forebrain. Dev Biol 296: 485-498, 2006.

6. Rajagopalan S, Deitinghoff L, Davis D, et al: Neogenin mediates the action of repulsive guidance molecule. Nat Cell Biol 6: 756-762, 2004.

7. Brinks H, Conrad S, Vogt J, Oldekamp J, et al: The repulsive guidance molecule RGMa is involved in the formation of afferent connections in the dentate gyrus. J Neurosci 24: 3862-3869, 2004.

8. Mirakaj V, Brown S, Laucher S, Steinl C, et al: Repulsive guidance molecule-A (RGM-A) inhibits leukocyte migration and mitigates inflammation. Proc Natl Acad Sci USA 108: 6555-6560, 2011.

9. Samad TA, Rebbapragada A, Bell E, et al: DRAGON, a bone morphogenetic protein co-receptor. J Biol Chem 280: 14122-14129, 2005.

10. Xia Y, Yu PB, Sidis Y, et al: Repulsive guidance molecule RGMa alters utilization of bone morphogenetic protein (BMP) type II receptors by BMP2 and BMP4. J Biol Chem 282: 18129-18140, 2007.

11. Shaw RJ, Liloglou T, Rogers SN, et al: Promoter methylation of P16, RARbeta, E-cadherin, cyclin A1 and cytoglobin in oral cancer: quantitative evaluation using pyrosequencing. Br J Cancer 94: 561-568, 2006.

12. Baba Y, Huttenhower C, Nosho K, et al: Epigenomic diversity of colorectal cancer indicated by LINE-1 methylation in a database of 869 tumors. Mol Cancer 9: 125, 2010.

13. Li VS, Yuen ST, Chan TL, Yan HH, et al: Frequent inactivation of axon guidance molecule RGMA in human colon cancer through genetic and epigenetic mechanisms. Gastroenterology 137: 176-187, 2009.

14. Rhodes A, Jasani B, Barnes DM, et al: Reliability of immunohistochemical demonstration of oestrogen receptors in routine practice: interlaboratory variance in the sensitivity of detection and evaluation of scoring systems. J Clin Pathol 53: 125-130, 2000 . 
15. Livak KJ and Schmittgen TD: Analysis of relative gene expression data using real-time quantitative PCR and the 2(-Delta Delta C(T)) Method. Methods 25: 402-408, 2001.

16. Niederkofler V, Salie R, Sigrist M, et al: Repulsive guidance molecule (RGM) gene function is required for neural tube closure but not retinal topography in the mouse visual system. J Neurosci 24: 808-818, 2004.

17. Yoshida J, Kubo T and Yamashita T: Inhibition of branching and spine maturation by repulsive guidance molecule in cultured cortical neurons. Biochem Biophys Res Commun 372: 725-729, 2008.

18. Monnier PP, Sierra A, Macchi P, et al: RGM is a repulsive guidance molecule for retinal axons. Nature 419: 392-395, 2002.

19. Matsunaga E, Tauszig-Delamasure S, Monnier PP, et al: RGM and its receptor neogenin regulate neuronal survival. Nat Cell Biol 6: 749-755, 2004.

20. Matsunaga E, Nakamura $\mathrm{H}$ and Chédotal A: Repulsive guidance molecule plays multiple roles in neuronal differentiation and axon guidance. J Neurosci 26: 6082-6088, 2006.

21. Matsunaga E and Chédotal A: Repulsive guidance molecule/ neogenin: a novel ligand-receptor system playing multiple roles in neural development. Dev Growth Differ 46: 481-486, 2004.

22. Halbrooks PJ, Ding R, Wozney JM, et al: Role of RGM coreceptors in bone morphogenetic protein signaling. J Mol Signal 2: 4, 2007.
23. Babitt JL, Huang FW, Wrighting DM, et al: Bone morphogenetic protein signaling by hemojuvelin regulates hepcidin expression. Nat Genet 38: 531-539, 2006.

24. Blanco Calvo M, Bolós Fernández V, Medina Villaamil V, et al: Biology of BMP signalling and cancer. Clin Transl Oncol 11: 126-137, 2009.

25. Piccirillo SG, Reynolds BA, Zanetti N, et al: Bone morphogenetic proteins inhibit the tumorigenic potential of human brain tumour-initiating cells. Nature 444: 761-765, 2006.

26. Hardwick JC, Van Den Brink GR, Bleuming SA, et al: Bone morphogenetic protein 2 is expressed by, and acts upon, mature epithelial cells in the colon. Gastroenterology 126: 111-121, 2004.

27. Lorente-Trigos A, Varnat F, Melotti A, et al: BMP signaling promotes the growth of primary human colon carcinomas in vivo. J Mol Cell Biol 2: 318-332, 2010.

28. Lombardo Y, Scopelliti A, Cammareri P, et al: Bone morphogenetic protein 4 induces differentiation of colorectal cancer stem cells and increases their response to chemotherapy in mice. Gastroenterology 140: 297-309, 2011.

29. Li J, Ye L, Mansel RE and Jiang WG: Potential prognostic value of repulsive guidance molecules in breast cancer. Anticancer Res 31: 1703-1711, 2011.

30. Esteller M: Cancer epigenomics: DNA methylomes and histonemodification maps. Nat Rev Genet 8: 286-298, 2007. 